\title{
Seismic energy radiation from dynamic faulting
}

\author{
Raul Madariaga \\ Laboratoire de Géologie, Ecole Normale Supérieure, 4 rue Lhomond, 75231 Paris Cedex 05, France
}

\begin{abstract}
Seismic radiation allows seismologists to probe the details of the rupture process during an earthquake. Waves recorded in the vicinity of the fault are used to invert for the details of rupture using a mixture of kinematic and quasi-dynamic methods. Seismic radiation is not only a tool to probe the earthquakes, it is also the essential dissipative mechanism by which ruptures exchange energy with the surrounding elastic media. Inversion has been used to study several earthquakes at relatively long wavelength, extending these results to higher frequencies requires improved understanding of the fundamentals of seismic energy balance which remain obscure, even if the fundamental theorems were established by Kostrov 30 years ago. In this paper we examine the simplest possible earthquake model, a propagating 2D antiplane crack and compute energy balance in detail. We show that energy can be expressed as simple integrals of energy release on the fault, that high frequencies are radiated by fast changes in rupture velocity and in jumps and kinks of the rupture front. We demonstrate that even if energy can be expressed as an integral of slip on the fault, it can not be localized on the fault.
\end{abstract}

\section{Introduction}

In spite of considerable progress in understanding earthquake dynamics, there are still a number of obscure point about elastic energy radiation and its role in controlling rupture. Recent studies have suggested that apparent energy release rate as measure from seismic radiation is of the order of several $\mathrm{MJ} / \mathrm{m}^{2}$, a huge number that probably reflects the fact that seismic rupture do not occur on a simple fault surface as modeled in laboratory experiments. Recently, Ide (2002) and Favreau and Archuleta (2003) showed that total energy release rate during the Imperial Valley and Kobe earthquakes was of the same order of magnitude as radiated energy and strain energy released by those earthquakes. This seems to confirm, the claim by Madariaga and Olsen (2001) that earthquakes propagate at an almost critical level so that the Griffith criterion for shear rupture is barely satisfied. This may explain why super-shear rupture is only observed in very special circumstances.

\section{Radiated Energy}

Kostrov (1964) defined radiated energy as :

$$
E_{s}=-\int_{0}^{\infty} d t \int_{S} \dot{u}_{i} \Delta \sigma_{i j} n_{j} d S
$$


integrated over a surface $S$ that surrounds the fault VERY far from it. Here $\Delta \sigma$ is the tensor of stress change produced by the seismic source and $u$ is the particle velocity in the far field of the source. So that effectively one can use the far field in it.

The correct expression for radiated energy in terms of fault slip and fault stress was given by Eshelby (1969) and Kostrov (1974), and has been used by Madariaga (1976), Ide (1992),Freund (1989), Favreau et al (2003) and many others. Kostrov's expression is :

$$
E_{s}=\frac{1}{2} \int_{\Sigma} \Delta \sigma_{i j} \Delta u_{i} n_{j} d S-\int_{\Sigma} G d S+\int_{0}^{\infty} d t \int_{\Sigma} \dot{\sigma}_{i j} \Delta u_{i} n_{j} d S
$$

where $E_{s}$ is the total radiated energy, $\Sigma$ is the fault surface and $\vec{n}$ is the fault normal, $\Delta u_{i}$ is the $i$ th component of the slip vector across the fault and $\Delta \sigma$ is the stress tensor change produced by slip. This expression is remarkable in that it expresses total radiated energy as a function only of changes in the state of stress and inelastic slip.

Very often this equation in the seismological literature this equation is integrated by parts to obtain :

$$
E_{s}=\frac{1}{2} \int_{\Sigma} \sigma_{i j} \Delta u_{i} n_{j} d S-\int_{\Sigma} \gamma d S+\int_{0}^{\infty} d t \int_{\Sigma} \sigma_{i j} \Delta \dot{u}_{i} n_{j} d S
$$

where $\sigma$ is the TOTAL stress acting on the fault. Although they are equivalent, equations (2) and (3) are very different and should not be used simultaneously in evaluating seismic radiation. In expression (3) the first term is the TOTAL strain energy change while in (2) the first term is just the self-energy change. Eshelby was the first to show that since the radiated field does not depend on absolute values of stress, radiated energy should not depend on absolute stresses either.

Recently Ide (2002) and Favreau and Archuleta (2003) used a version of (2) in order to compute the radiation from several earthquakes for which slip on the fault had been computed by inversion of near field seismograms. In these inversions. Although this inversion technique is only valid at relatively low frequencies, the slip distributions are robust. From the slip distribution a stress change field is computed so that expression (2) can be computed term by term. Many authors have expressed doubts that one can decompose $E_{s}$ into individual contributions by fault elements. We will examine this problem in detail in the following.

\section{Radiation from a simple antiplane fault}

For simplicity I will study the semi-infinite mode III crack shown on Fig 1 that extends along the negative $x$-axis and is in equilibrium for $t<0$. At $t=0$ rupture starts moving at constant speed $v_{r}$ along the positive $x$ axis. The stress intensity factor at the rupture tip at $x=0$ is noted $K_{0}$ and the corresponding quasi-static energy release rate is $G_{0}=K_{0}^{2} /(2 \mu)$. Radiation from this semi-infinite fault can be computed by Kostrov's (1966) solution for a flat crack (see, e.g. Madariaga, 1977, 1984) or Adda-Bedia and Arias (2004) who studied the more complex problem of radiation from a fault that kas a kink at the origin.

From Madariaga (1977) we know that rupture of the semi-infinite fault produces a shear wave front with a particle velocity jump:

$$
\dot{u}(r, \theta, t)=\frac{K_{0}}{\mu} \frac{v_{r}}{\sqrt{2 \pi r}} \frac{\sin \theta / 2}{1-v_{r} / \beta \cos \theta} H(t-r / \beta)
$$


at an observer situated at coordinates $r, \theta$. Here $\mu$ is the elastic constant and $\beta$ the shear wave speed. This is what seismologists call a far field solution, i.e. the dominating term as $r \longrightarrow \infty$. Since particle velocity is constant behind the rupture front in (2), the total energy radiated by this fault is unbounded and the computation of seismic energy as defined by Kostrov does not make sense.

\section{Radiation from a finite mode III fault}

In order to compute seismic energy we let the fault of section (1) run for a short length Lalong the $x$ axis and then stops. When it stops it radiates a wave of the same form a (2) but with rreplaced by Fraunhoffer's approximation

$$
r^{\prime}=\sqrt{(x+L)^{2}+y^{2}} \approx r(1+L / r)
$$

The far field wave generated by the finite rupture episode is then:

$$
\dot{u}(r, \theta, t)=\frac{K_{0}}{\mu} \frac{v_{r}}{\sqrt{2 \pi r}} \frac{\sin \theta / 2}{1-v_{r} / \beta \cos \theta}\left\{H(t-r / \beta)-H\left[\left(t-r / \beta-L / v\left(1-v_{r} / \beta \cos \theta\right)\right]\right\}\right.
$$

Using (1) we can compute radiated energy exactly for this model. We get

$$
E_{s}=\int_{0}^{\infty} d t \int_{S} \frac{\mu}{\beta} \dot{u}^{2} d S
$$

which gives after a change in the order of integration and use of (5):

$$
\begin{aligned}
E_{s} & =\frac{K_{0}^{2}}{\mu} \frac{v_{r}}{\beta} \frac{1}{2 \pi} \int_{0}^{2 \pi} \frac{\sin ^{2} \theta / 2}{1-v_{r} / \beta \cos \theta} d \theta \\
& =\frac{K_{0}^{2}}{2 \mu} L\left[1-\sqrt{\frac{1-v_{r} / \beta}{1+v_{r} / \beta}}\right] \\
& =G_{0} L-G\left(v_{r}\right) L
\end{aligned}
$$

Radiated energy is the difference between the quasi-static and dynamic work of the stress concentration $K_{0}$ as it moves a distance $L$ along the $x$ axis.

We can now generalize (7) and write that total energy for an arbitrarily moving rupture is

$$
E_{s}=\int_{0}^{L}\left(G_{o}(x)-G(v, x)\right) d x
$$

a similar result was obtained by Freund (1989) by a modification of Kostrov's equation (2).

\section{Fault slip and energy distribution.}

The most important question from a seismological point of view is whether (8) or one of its variations can be used to back trace the source of energy from a seismogram to the fault. We would like to know whether the energy observed at time $t$ at the observer comes from an specific point on the fault. For displacement and particle velocity, this problem was already studied in some detail by Bernard and Madariaga (1984) in the 3D case and by Madariaga (1984) in 2D. Following these authors the field observed at time $t$ at an observer situated 
at the cylindrical coordinates $(r, \theta)$ comes from an isochrone, a curve on the fault for $2 \mathrm{D}$ faults or a few isolated point for a $1 \mathrm{D}$ fault. If the latter problem can be solved, then the computation of energy flow as a function of time and position can be computed.

Unfortunately, radiated energy can not be decomposed in the same way, because radiated energy is averaged on the focal sphere and arrival times depend on the azimuthal angle and distance. Thus, the computations by Ide (2002) and Favreau and Archuleta (2003) can be considered as reasonable estimates of the contribution of points on the fault to total energy balance, but they can not be decomposed into individual contibutions of different segments of the fault, unless rupture history is well resolved. The main reason is that fault radiation is not simply proportional to slip, it includes effects of diffraction (and refraction for in-plane problems) by the tip of the fault and other geometrical or mechanical discontinuities.

\section{Generalization}

We presented above the solution for the motion of straight antiplane shear fault. Similar results can be obtained for plane shear using the solution by Madariaga (1977) although finding an expression similar to (7) is much more difficult. Recently Adda-Bedia and Arias (2004) have found a general solution to the problem of Fig 1 where the fault starts moving along a kinked fault. using their results Adda-Bedia and Madariaga (in preparation) found that the expression (7) is still valid if the stress intensity factor is replaced by a modified value that takes into account the effect of the kink on the stress field in the vicinity of the crack tip. Extension of these results to 3D ruptures is much more difficult, except when rather strong kinematic assumptions are made as in the case of a circular crack propagations at constant speed (Madariaga, 1976; Ide, 2002)

\section{Conclusions}

We have shown that seismic radiation can be computed exactly from the slip change that occurs on the fault. There are several important caveats that have to be carefully considered, but in principle at least the computation is feasible. The main difficulty is not so much computing energy, but identifying the place on the fault where energy comes at certain time. This requires solving a non-linear back-projection problem when rupture velocity is known for every point on the fault. In reality, determining the rupture velocity is a much more difficult problem that computing the distribution of slip, so that I expect that much better rupture invert-ions will be required before we can fully identify the origin of radiation on the fault.

\section{References}

[1] Adda-Bedia, M., J. Mech. Phys. Solids, 52, 1407-1420, 2004.

[2] Adda-Bedia and R.Arias, J. Mech. Phys. Solids, 51, 1287-1304, 2003.

[3] Eshelby, J.D., J. Mech. Phys. Solids, 17, 177-197, 1969.

[4] Favreau, P. and Archuleta, R., Geophys. Res. Lett., 30, 1198, 2003. 
[5] Freund, L.B. , Dynamic Fracture Mechanics, Cambridge Univ. Press, New York, 1989.

[6] Ide, S., Bull. Seismol. Soc. Am. , 92, 2994-3005, 2002.

[7] Kostrov, B.V., Appl. Math. Mech., 30, 1241-1248, 1966.

[8] Kostrov, B.V., Izv. Earth Physics, 1, 23-40, 1974.

[9] Madariaga, R., Bull Seismol. Soc. Am., 66, 639-666, 1976.

[10] Madariaga, R. Geophys. J. Roy. astr. Soc., 51, 625-651, 1977.

[11] Madariaga, R. Annales Ggeophysicae, 1, 17-23, 1983.

[12] Madariaga, R. and K.B. Olsen, Pageoph, 157, 1981-2001, 2000.

\section{Figure}

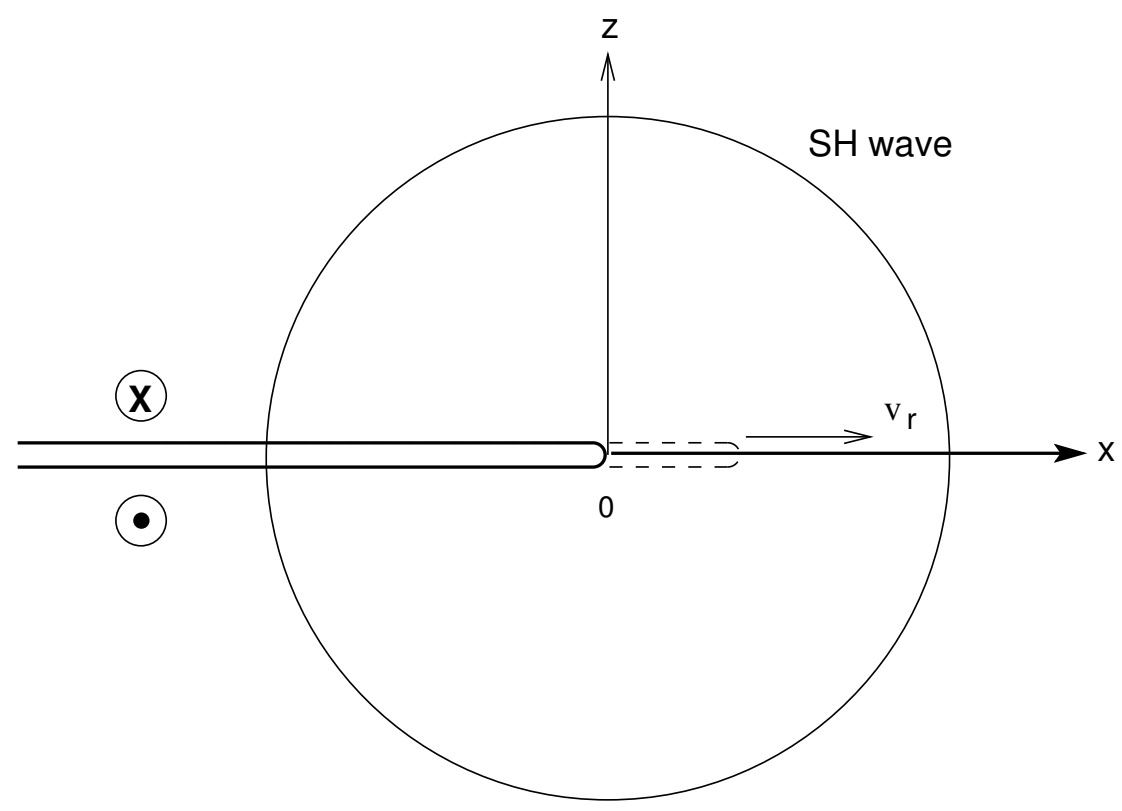

Fig 1. Simple model of a planar antiplane fault that suddenly extends along the $x$-axis at constant speed $v_{r}$. This is one the rare crack radiation problems that can be fully solved exactly 